\title{
Research on Blind Box and its Marketing Strategy
}

\author{
Ziwei Zhang ${ }^{1, *}$ \\ ${ }^{1} X i$ 'an Gaoxin No.1 High School, Xi'an Shaanxi China 710000 \\ *Corresponding author. Email:854267509@qq.com
}

\begin{abstract}
With the arrival of Generation $\mathrm{Z}$ and the rapid update and iteration of entertainment ways, the blind box industry is also increasingly prosperous. Blind boxes are small, but they are making a big splash in the market. Even created a set of special marketing methods and economic principles, known as the "blind box economy". People keep the excitement of buying blind boxes instead of feeling tired. It is apparent that it can not be explained by traditional economics which considers everyone as a rational person. This paper is going to change the angle of analysis of the crazy behavior of buying blind box, from the point of view of consumer psychology. And also combine with the traditional economics principle to study the marketing strategy of producers. The deep analysis of the blind boxes economy will be conducted on the basis of the existing consumer psychology principles and the data of blind boxes. The psychology theorems are from Skinnerian theory, gambling effect, the effect of the sheep flock. And it can be concluded that the marketing strategy is based on the price strategy, the use of intellectual property rights, hunger marketing and distribution channels.
\end{abstract}

Keywords: blind box economy, consumer psychology, marketing strategy, COVID-19.

\section{INTRODUCTION}

With the born of "Generation Z", more and more people are crazy about the blind boxes. Blind box stemmed from Japan. Just as its name implies, they are boxes that may contain surprise or disappointment. Each series has twelve same external packing boxes, and every box contains different toys. Buyers don't know which they buy until they open the box. Seller put the most special one called secret or hidden box in each series. However, the possibility to get the secret one is $1 / 144$. Thus, many blind box collectors spend heavily in order to get the secret toy. Under the premise of rational economic man, the marginal benefit is diminishing, buying an additional unit of product will cause the additional benefit to decrease. Whereas, for consumers of blind boxes, the more they buy, the more they want. The diminishing marginal utility theory seems not available to the blind box economy. What is the reason? Why do the followers of the small boxes spend a lot of money on them? What is their psychology? What is the marketing strategy of the sellers? The following content will first talk about what kind of psychology the blind box satisfies consumers, and besides, it will analyze the marketing strategy of the blind box.

\section{ANALYSIS ON THE BLIND BOX}

Next, this paper will discuss the blind box from the perspective of behavioral economics.

\subsection{Skinner Box}

Skinner Box is a laboratory apparatus used in the experimental analysis of behavior to study animal behavior. There is a mouse in the box, and there is a lever on the inner wall of the box. When the mouse pushes the lever once or more times, food outside the box will drop in randomly. Thus, the mouse learns to push the lever and get food. The conclusion is that, just repeat the behavior and the reward over and over again, there will be a connection between the two, and rewards play a positive reinforcement role. Applying the theory on the blind box, if buyers get a nice toy, they will feel excited, the good luck will push them to buy more. If they don't get the one they like, they may keep buying until they get the one they like. This buying behavior is like the mouse in the box, pushing the lever uninterruptedly, even if no food drops in. Unremitting push equals continuous opening blind box. The random drop of food equals the secret or nice toy in the box. The lure of the unknown motivates consumers to buy again and again. There appears a strong connection or 
conditioned reflex between buying blind box and picking the most special one.

\subsection{Love is blind (Gambling effect)}

Compared with other Garage Kit(toys, models can be collected by the players), blind box is much hotter in recent years. Research has shown that blind box is the easiest approach for the new players to fall in love and become loyal customers. One of the most popular blind box brands named POP MART generated 8.18 billion yuan in sales in the first half of 2020[1]. What brings such a preference of the players? For the consumers of blind boxes, the most enjoyable and stimulating moment is the time to open the boxes. Along with tension, excitement, anticipation. "blind" makes the trip of the consumption further and further. In fact, buying blind boxes is similar to gambling. Adopting the uncertainty of the consequences, improve the sense of thrill on buying, and make consumers have the desire to repeat the purchase. If blind boxes are not blind any more, consumers can know which toys in the boxes. Thus, they will only choose their preferences among the twelve toys. After buying some of them, they will stop the consumption. Whereas, in the procedure of consuming blind boxes, consumers not only pursue the objects in the boxes, but also the process of consideration, entanglement, and excitement. At the same time, the uncertainty of the blind boxes also brings consumers a sense of security before opening the boxes, even the toys inn the boxes are not their favored. The unknown arouses interests, and unknown makes addiction.

\subsection{The Effect of Sheep Flock and social needs}

The Effect of Sheep Flock means that one's behavior is influenced by others, and causes conformity. In the market, the Effect of Sheep Flock is common. For instance, when people buy stocks the first time, they often follow the people around them who just made a profit. The new buyers will buy the same stock with them. Blind box is the same. The plain little boxes aren't magnetic enough to attract passengers to buy them. Thus, for the players of blind boxes, their first try might because of the others' behavior of buying blind boxes. Even some players reflex that, when some new blind boxes come out, they don't feel how special they are. However, as more and more players post the toy on social platforms, they will also find the "normal toy" to look better and better. When you place a single toy on the shelf, it may not be impressive. But when you see a wall filled with the toys, it must stimulate the wants to own all of the blind boxes. During the "Generation Z", the social platform becomes more and more popular. Xiaohongshu is the hottest one. People always follow the recommendation of the bloggers. People on this platform always share their life or their favorite goods.
Users can exchange their ideas through remarking under publishers' plogs or vlogs. When someone first sees the toys wall of the blogger, that must be a big surprise to push him or her to purchase the blind box. Senior players also teach the strategy to pick blind boxes according to their sound of shaking the box, and the weight of different toys. These give more confidence and support to new players to buy. Additionally, the blind box also meets the needs that change over time.

\section{THE MARKETING STRATEGIES OF PRODUCERS}

\subsection{Price attraction.}

According to estimates, by 2024, the global fashion toy market scale will reach $\$ 448$ billion dollars.[2] By 2019 , in terms of retail value, Pop Mart is China's largest fashion toy brand, the market share was $8.5 \%$ and recorded rapid growth.[3] One of the attractions of Pop Mart to consumers is the price. Most of the blind boxes are 59 yuan each. And it also sells bigger fashion toys which price is around 299-399 yuan. According to the research of price acceptance of China's Generation $\mathrm{Z}$ in $2020,27.8 \%$ of people would like to consume between $51-100$ yuan, and $31.6 \%$ of consumers choose 101-500 yuan[4]. The price of most blind boxes is set in the areas where consumer acceptance is highest. The price strategy can also be considered as "Mantissa Pricing". The Mantissa Pricing strategy refers to that when determining the retail price, it makes use of consumer's psychology of seeking cheap prices to set non-integer prices, ending with odd numbers. Thus, the price of blind boxes is always ended with 9. In the strategy of a lower price, the more expensive fashion toy will be the reference object of the blind box, for example Bearbrick. Bearbrick is one of the popular fashion toys with high prices, the same size as bearbrick is ten times more expensive than the blind box.

\subsection{IP system}

The core strategy of blind boxes is IP. IP is the carrier medium of users' emotions. It can be content in fields such as literature, music, animation, drama, movie and games. An excellent IP needs good content to gather fans and users, and cultivate users' emotion through content. And finally realize the commercial value of IP. Actually, all fashion toys are derivatives of IP. Like the peripheral products of Disney are the derivatives of Disney culture. IP has a pivotal role in the whole market, the IP licensing industry has huge growth opportunities in China. Derived by continued growth in demand, the market size of China's IP licensing industry is forecast to reach 168.6 billion yuan by 2024 , with a CAGR of 14.8\% from 2019.[5] By 2020 June 30, Pop Mart has operated a total of 93 IPs, including 12 propriety IPs, and 56vnon-exclusive IPs. Through 
signing a contract with Molly IP, Pop Mart became the most popular blind box brand. Later, Pop Mart cooperated with many other famous IPs, such as Hello Kitty, Mickey and Minnie. Pop Mart made full use of IP marketing strategy, and quickly captured the market share. Completed IP captivate the hearts of consumers. Female players of blind boxes account for $75 \%$, with young people aged 18-29 years old(58\%) as the main consumer group.[6] Students are the main consumers of blind boxes. Blind boxes create common topics for the young generation. There are many fans of blind boxes who share their experience of picking blind boxes, and exchange the toys on some platforms like Xianyu with each other. Thus, blind boxes have strong social attributes and entertainment properties. The purchase of the blind boxes often attracts young people to buy in groups.

\subsection{Hunger Marketing}

Hunger Marketing refers to producers decrease the number of products on purpose in order to create the illusion that demand exceeds supply, and maintain the image of the product and current price. In consumer psychology, economists consider "A thing is values if it is rare"the price of commodities will rise due to scarcity. For example, before buying a house, pay sincerity money first. There seems to be a long line waiting to buy the house. In fact, it is just the real estate companies who want to arouse the hunger of consumers and arouse their inner impulse to buy the house. That is, because of "scarcity", housing prices are higher and higher. The producers of the blind box also make use of hunger marketing. Every series has 12 normal kinds of toys and a secret toys. Without doubt, the secret toy is the best representation of scarcity. Consumers not only pay more attention to it, but also are willing to spend more on it. Some players of blind box spend thousands of yuan in order to buy the secret toy which is 59 yuan. Thus, a profession who drives up the market price was born-scalpers. In offline stores, scalpers will judge the boxes by shaking them and feeling the weight of the boxes. They will then buy popular and secret toys and resell them on secondary platforms, usually at a higher price than the retail price.

\subsection{Diversity of distribution}

The distribution channels of blind boxes are offline stores, vending machines, e-commerce, second-hand platform, fashion toy exhibition. Different kinds of ways to buy blind boxes will bring consumers various feelings. For the entity stores, consumers choose the blind box depends on their experience, skills and luck. It is more common for mature players to buy, and have a higher possibility for them to get what they want with their consumption experience. Whereas, some players choose to buy from the vending machines. Buying the toys you like from the machine can only depend on your luck. What buyer only needs to do is to decide which series they want, and pay for it, and the vending machine will choose the blind box automatically. For the online distribution, such as the mini program in WeChat app, it will bring consumers another kind of consumption experience. Buyers not only can shake the phone seems like they buy at the entity store, but consumers can also choose to use hint cards (automatically exclude impossible toys) and display cards that need additional payment. Such a kind of card gives the consumers instant gratification in the entity store. Pop Mart also employs a membership point system. The purchase amount is automatically converted into points, which can be redeemed not only for gifts but also for the online mini-program hint cards. The invisible connection between online and offline stores has been set up. The cost of the transportation of the blind box is low, and online channels satisfy the social need of players. Because of the COVID-19, online shops and the vending machine began to take more proportion of the channels of a blind box. The future of blind box sales will be offline and online integration of development.

\section{CONCLUSION}

For the blind box economy, it is unreasonable to only consider the value of the blind box. The hypothesis of rational economic people is obviously not suitable to the blind box economy. It may have a better explanation of why consumers are such crazy about buying blind box which doesn't support the diminishing utility. This paper analyzes the blind box economy and explores the marketing strategy of Pop Mart. From the perspective of consumer psychology, the Skinner box effect. It can be obtained that consumers are like the mice in the box to do the specific action and get rewards. Finally, purchasing blind boxes became a prerequisite for getting the secret toy. Additionally, the gambling effect makes players enjoy the excitement during the process of opening the boxes. The effect of the sheep flock can be apparently concluded because of the consumers' social needs.

Price attraction, comparably lower than those expensive fashion toys and the non-integer price attract a lot of consumers. Besides, using IP is the core of blind box market, make full use of the image of the original IP and the love of their fans. Hunger marketing introduces scarcity of the blind box, not only stimulate the irrationality of consumers but also rise the prices. Various distribution channels bring consumers different experiences of consumption. Although the arrival of COVID-19 has hit the sales of offline stores, it has provided a good development opportunity for online sales. Several psychological effects of consumers bring new perspectives of studying the blind box economy. 


\section{ACKNOWLEDGMENT}

Over the course of my research and writing this paper, I would like to express my thanks to all those who have helped me.

First, I would like to express my gratitude to all those who helped me during the writing of this thesis. A special acknowledgement should be shown to Professor Zhang juanjuan, from whose marketing class benefited greatly, I am particularly indebted to assistant teachers who gave me kind encouragement and useful instruction all through my writing.

Sincere gratitude should also go to all my learned Professors and warm-hearted teachers who have greatly helped me in my study as well as in my life.

And my warm gratitude also goes to my friends and family who gave me much encouragement and financial support respectively. Moreover, I wish to extend my thanks to the library and the electronic reading room for their providing much useful information for my thesis.

\section{REFERENCES}

[1] Prospectus of Pop Mart International Group Limited POP MART 2020 Prospectus (2020: 5-7)

[2] Frost\&Sullivan data.iimedia,cn Global trend toy industry market size and forecast in 2015-2024

[3] iiMedia Research data.iimedia.cm Market Size of IP Licensing Market in China 2015-2024

[4] CY337 Analysis of the current situation and prospect of the development of China's trendy toys in 2020: the market scale is expected ti reach tens of billions of dollars www. Chyxx. Com 2020.6: the 1 page

[5] Xinyue Han From the perspective of behavioral economics to see the secret of blind box China academic journal electronic publishing house (2019:1-2)

[6] Muhui Su When blind box meets IP consumption China academic journal electronic publishing house (2019:1-3)

[7] Huaile Qi, The marketing strategy analysis on the basis of Z Generation's consumption psychology _ Using blind box as an example China academic journal electronic publishing house, 2020:2-3) 